\title{
Der innenpolitische Reformprozeß in Japan
}

\author{
Von Heinrich Kreft ${ }^{1}$
}

\section{Das Ende der Ein-Parteien-Dominanz}

Im Sommer 1993 verdrängte in Japan eine Koalition aus acht Parteien die konservative Liberal-Demokratische Partei (Jimin-to, LDP) nach 38 Jahren erstmals von der Regierungsverantwortung. Zwischen einem erfolgreichen Mißtrauensvotum am 18. Juni und der Amtsübernahme der neuen Regierung unter Ausschluß der LDP am 9. August wurde das über Jahrzehnte stabile Parteien- und Machtgefüge Japans aus den Angeln gehoben. Bis heute sind die Veränderungen noch nicht zu einem vorläufigen Ende gekommen. Eine Weichenstellung wird von den nächsten Unterhauswahlen erwartet, die spätestens im Sommer 1997 stattfinden.

Die LDP war 1955 aus dem Zusammenschluß mehrerer konservativer Parteien hervorgegangen. Vom Zeitpunkt ihrer Gründung bis zum August 1993 stellte die LDP ohne Unterbrechung die Regierung, in der alle Parteipräsidenten quasi automatisch auch das Amt des Premierministers übernahmen. Noch vor der LDP hatten sich ebenfalls 1955 mehrere sozialistische Parteien zur Sozialistischen Partei Japans (Nihon shakai-to, SPJ) ${ }^{2}$ zusammengeschlossen. Der SPJ, als mit Abstand zweitgrößter Partei, blieb 38 Jahre lang nur die Oppositionsrolle. Diese stabile Rollenverteilung zwischen LDP und SPJ, auch "1955er System" genannt, ging im Sommer 1993 zuende.

\section{Die Reformunfähigkeit der LDP}

Die LDP konnte sich 38 Jahre im wesentlichen ungefährdet an der Regierung halten. Japan entwickelte sich in dieser Zeit dank eines schnellen wirtschaftlichen Aufstiegs aus den Trümmern seiner totalen Niederlage im "Großen Pazifischen Krieg" zu einer führenden Industrie- und Finanzmacht. Die LDP konnte sich dabei auf eine fähige Bürokratie und die Großindustrie stützen, mit denen sie das viel zitierte "Eiserne Dreieck" bildete. Diese enge

\footnotetext{
Der Beitrag gibt die persönliche Meinung des Verfassers wieder.
} 
Interessenverquickung führte jedoch seit den 70er Jahren zu wachsendem Filz und Korruption.

Die Anfänge des japanischen Parlamentarismus gehen auf die Meiji-Verfassung von 1889 zurück. Die zweigeteilte Form des heutigen Parlaments in Ober- und Unterhaus basiert auf der Verfassung von 1946. Seit der Demokratisierung durch die amerikanische Besatzungsmacht konnten das Parlament und andere durch Wahlen legitimierte Institutionen nie den Einfluß erlangen wie in vergleichbaren westlichen Demokratien. Die Macht lag vielmehr in einem schwer durchschaubaren Beziehungsgeflecht von LDP, Ministerialbürokratie und der Großindustrie. Schwächster Teil des Dreiecks war dabei die LDP, deren Politiker finanziell von der Wirtschaft und bei den Regierungs- und Gesetzgebungsarbeiten in hohem Maße von der Ministerialbürokratie abhängig waren. Die Opposition hatte indes zu keiner Zeit eine realistische Möglichkeit, die LDP von der Regierung zu verdrängen. Ihre Autorität wurde erst durch eine ganze Kette von Korruptionsskandalen untergraben, in die wiederholt führende LDP-Mitglieder verwickelt waren.

1976 mußte Premierminister Kakuei Tanaka wegen seiner Verstrickung in den LockheedBestechungsskandal zurücktreten. Er wurde schließlich zu einer mehrjährigen Haftstrafe verurteilt, blieb aber dennoch bis zu seinem Tode 1993 eine hinter den Kulissen agierende einflußreiche Parteigröße. Obwohl die LDP im Sog dieser Affäre einen Tiefpunkt im öffentlichen Ansehen erreichte, konnte die Opposition in den folgenden Wahlen dennoch keinen Regierungswechsel herbeiführen.

In den 80er Jahren verloren im Zuge des Recruit-Cosmos-Bestechungsskandals fast 50 Politiker vor allem der LDP, aber auch der Oppositionsparteien ihre Positionen, darunter Premierminister Noburo Takeshita und Finanzminister Kiichi Miyazawa. In den Oberhauswahlen von 1989 büßte die LDP ihre absolute Mehrheit in der zweiten Kammer ein. Diese Wahlniederlage und die Belastung wichtiger Parteiführer durch den Recruit-CosmosSkandal führte Ende 1989 zur Wahl des reformorientierten Toshiki Kaifu zum Parteivorsitzenden und damit Premierminister. Der als "sauber" geltende Kaifu, der als Mitglied der kleinsten LDP-Faktion aber über keine bedeutende Hausmacht verfügte, setzte sich von Anfang an für innenpolitische Reformen ein, stieß damit allerdings sofort auf den erbitterten Widerstand der alten Parteibarone.

\section{Die Notwendigkeit politischer Reformen}

"Politische Reform" ist ein Schlüsselbegriff, den seit den frühen 70er Jahren viele japanische Politiker im Munde führten, und es darf unterstellt werden, daß viele spätestens seit dem Recruit-Cosmos-Skandal auch von ihrer Notwendigkeit überzeugt waren. Bereits Premierminister Takeshita hatte Ende 1988 den damaligen Vorsitzenden des LDP-Wahlaus- 
schusses, Masaharu Gotoda, mit Untersuchungen zu möglichen Reformen beauftragt. Im Mai 1989 legte der LDP-"Ausschuß für politische Reformen" Premierminister Takeshita einen "Rahmenplan" vor, der jedoch erst von Premierminister Kaifu aufgegriffen wurde.

Die LDP war seit ihrer Gründung in hohem Maße von Spendengeldern der Wirtschaft abhängig. Aufgrund hoher Wahlkampfkosten, niedriger Mitgliedsbeiträge und fehlender Wahlkampfkostenerstattung aus Steuergeldern war der Finanzbedarf der LDP und ihrer Abgeordneten immer sehr hoch. Statt eines Parteienfinanzierungsgesetzes gab es nur ein "Spendengesetz". Der überwiegende Teil der Spendengelder floß jedoch unkontrolliert an die Partei, ihre Mandatsträger und Kandidaten sowie vor allem an die Führer der parteiinternen Faktionen ${ }^{3}$. Der Geldbedarf eines japanischen Politikers, der sich um ein Mandat bewirbt, war immer um ein vielfaches höher als in Europa.

Der Wahlerfolg eines Kandidaten basiert traditionell im wesentlichen auf den sog. "Sanban", den drei "Ban": eine eigene Hochburg ("Chiban", ein bekanntes Gesicht ("Kanban") sowie wohlgefüllte und offene Taschen für substantielle Wohltaten im eigenen Wahlkreis ("Kaban"). Zur Erfüllung der gegenseitigen Verpflichtungen zwischen Politikern und ihren Wählern wie auch vor allem zwischen Politikern selbst ist ein ständiger hoher Geldfluß vonnöten. Der Einfluß der Faktionsführer in der LDP stützt sich auf die Zahl ihrer Gefolgsleute in Ober- und Unterhaus, die sie durch finanzielle Großzügigkeit an sich binden. Den größten Teil ihrer Wahlkampfgelder erhalten die einzelnen Abgeordneten nicht von der Parteizentrale, sondern von ihrem jeweiligen Faktionschef. Aufgrund des hohen Stellenwerts der Spendenakquisition suchen die Parteibarone engste Kontakte zur Wirtschaft und ihren Verbänden. Hierin liegt einer der Hauptgründe für die Korruptionsanfälligkeit hoher LDP-Politiker, die schließlich zum Niedergang der Partei führten.

Auch das Wahlsystem aus Mehrheitswahl mit Mehrsitzwahlkreisen wurde für die politische Krise verantwortlich gemacht. Die 511 Sitze des Unterhauses wurden bis 1993 in 129 Mehrsitzwahlkreisen mit jeweils 2 bis 6 Mandaten vergeben. ${ }^{4}$ Wollte die LDP mehr als 129 Mandate erringen, mußte sie in der Mehrzahl der Wahlkreise mehr als einen Kandidaten aufstellen, die zwangsläufig auch gegeneinander antraten und i.d.R. von unterschiedlichen Faktionen der Partei unterstützt wurden. So war es nicht verwunderlich, daß die Wahlkämpfe weniger durch (partei-)programmatische Auseinandersetzungen bestimmt wurden, als durch den persönlichen und finanziellen Einsatz einschließlich politischer Zusagen von

Die Parteigruppierungen der LDP werden allgemeinhin als "habatsu" bezeichnet, was im Englischen mit "faction" übersetzt wird. Im Deutschen hat sich dafür die Übersetzung "Faktion" eingebürgert im Unterschied zu den "Fraktionen" als Gruppen von Abgeordneten einer Partei im Parlament.

4

Einzige Ausnahme war die Inselgruppe Amani, die einen eigenen Wahlkreis mit nur einem zu vergebenden Mandat bildete. 
Vergünstigungen für bestimmte lokale Interessengruppen. Kennzeichen der japanischen Wahlen war nicht so sehr der Wahlkampf zwischen Kandidaten der Regierung und der Oppositionsparteien, sondern zwischen offiziellen LDP-Bewerbern verschiedener Faktionen und "unabhängigen" konservativen Kandidaten. Letztere traten im Erfolgsfall zumeist in die LDP ein und konnten bei folgenden Wahlen als offizielle Kandidaten der Partei antreten.

In der japanischen "Konsensdemokratie" steht nicht der harte Schlagabtausch zwischen politischen Gegnern im Vordergrund, sondern das sorgfaltige Austarieren unterschiedlicher Meinungen und Strömungen. Kleine pragmatische Ziele stehen anstelle von programmatischen Grundsätzen und großen Visionen. Der Wahlmodus und die Regelung der Spendenpraxis standen von Anfang an im Zentrum der Diskussion um politische Reformen. Insbesondere die Schaffung von fairen und transparenten Regeln für die politische Spendenpraxis gefährdete jedoch die Finanzquellen und damit auch den Einfluß der LDP-Faktionschefs.

Kaifu, der die bis dahin höchsten Popularitätswerte eines amtierenden Premierministers erzielte, wurde im Oktober 1991 von den "Königsmachern" der LDP zum Rücktritt gezwungen. Seine Reformentwürfe gelangten nicht einmal in die Ausschußberatungen des Parlaments. Die Wahl Kiichi Miyazawas zu seinem Nachfolger bedeutete für die Reformkräfte in der LDP einen Rückschlag. Mit dem ehemaligen Finanzminister, der nur zweieinhalb Jahre zuvor wegen seiner Verstrickung in den Recruit-Cosmos-Skandal hatte zurücktreten müssen, kehrte ein Exponent der alten reformunwilligen Garde an die Spitze von Partei und Regierung zurück. Formell verfolgte auch Miyazawa das Ziel politischer Reformen weiter, doch mit deutlich geringerem persönlichen Einsatz. Neue Bestechungsskandale insbesondere um das Speditionsunternehmen "Sagawa Kyubin" setzten die Regierung unter Druck. Prominentestes Opfer dieses neuen Skandals war Shin Kanemaru, der Patriarch der dominierenden Takeshita-Faktion, die schließlich in der Auseinandersetzung um seine Nachfolge zerbricht.

\section{Juli-Revolution oder konservative Selbsterneuerung?}

Verärgert über die dilatorische Behandlung der von der Bevölkerung gewünschten politischen Reformen durch die Regierung, stimmte am 18. Juni 1993 die Hata-Ozawa-Gruppe, der kleinere Teil der ehemaligen Takeshita-Faktion, für einen Mißtrauensantrag der Opposition. Zum ersten Mal in ihrer Geschichte war die LDP damit ohne Mehrheit im Unter-

Vgl. Manfred Pohl, Hintergründe einer "Einparteien-Demokratie": Die Anatomie der japanischen Regierungspartei, in: Ulrich Menzel (Hrsg.), Im Schatten des Siegers: Japan Staat und Gesellschaft, 2. Band, Frankfurt a.M., 1989, S. 296. 
haus. ${ }^{6}$ Premierminister Miyazawa löste daraufhin das Parlament auf und schrieb Neuwahlen aus. Insgesamt 46 Abgeordnete traten aus der LDP aus und gründeten die zwei neuen Parteien Shinseito ${ }^{7}$ und Sakigake ${ }^{8}$. Bereits im Mai 1992 hatte der ehemalige LDP-Abgeordnete und Gouverneur Morihiro Hosokawa die "Neue Japan Partei" (JNP) ${ }^{9}$ gegründet und sich ganz der Reformierung des politischen Systems verschrieben.

Die Juli-Wahlen selbst brachten keine wesentlichen Überraschungen. Die LDP konnte den Exodus nicht kompensieren und die absolute Mehrheit zurückgewinnen, blieb aber mit Abstand stärkste Partei. Der große Verlierer war die Sozialistische Partei, die nahezu die Hälfte ihrer Mandate einbüßte zugunsten der JNP und den zwei LDP-Abspaltungen. Nicht die traditionelle Opposition, sondern eine neue "Rechte" profitierte damit vom Niedergang der LDP. Die drei neuen konservativen Parteien blieben allerdings aufgrund des stark persönlichkeitsbezogenen Wahlverhaltens der Bevölkerung weit hinter der Mandatszahl der LDP zurück. Schließlich gelang es den konservativen LDP-Renegaten jedoch gemeinsam mit den Sozialisten, die 38 Jahre lang eine Fundamentalopposition betrieben hatten, und weiteren kleinen Oppositionsparteien eine Anti-LDP-Koalition zu schmieden. Morihiro Hosokawa wurde Anfang August 1993 zum ersten Nicht-LDP-Premierminister seit 1955 gewählt.

\section{Die "politischen Reformen" als vorrangiges Ziel der neuen Regierung}

Premierminister Hosokawa ließ von Anfang an keinen Zweifel daran, daß die Reform des politischen Systems wichtigstes Ziel seiner Regierung war. Kernstück der Reformen sollte die Ablösung der bisherigen Mehrheitswahl mit Mehrsitzwahlkreisen durch ein Mischsystem aus Mehrheitswahl in Einerwahlkreisen und Verhältniswahl werden. Die Regierungsparteien einigten sich auf eine Reduzierung der Unterhausmandate von 511 auf 500. Davon sollte jeweils die Hälfte in Direkt- und Verhältniswahl mit nationaler Liste vergeben werden, mit jeweils zwei Stimmen pro Wähler. Die LDP, die sich nun ebenfalls für Einerwahlkreise und ein Mischwahlsystem aussprach, trat dagegen für eine Reduzierung der Mandate auf 471 ein (300 nach Mehrheits- und 171 nach Verhältniswahl) sowie für eine Stimme pro Wahlberechtigten. Dieser Gegenentwurf der LDP war dabei weniger das Resultat eigener Überzeugungen als vielmehr der Versuch, die Koalitionsregierung zu spalten. Ein Mehrheitswahlsystem kombiniert mit Einerwahlkreisen favorisiert eindeutig

Abgesehen von einer kurzen Periode Anfang der 80er Jahre, als sie eine Koalition mit Abgeordneten eingehen mußte, die die Partei vorübergehend verlassen hatten. übersetzt.

8 Sakigake ins Deutsche zumeist mit "Vorbote", ins Englische mit "Harbinger" übersetzt.

9

Englisch: Japan New Parte (JNP). 
die stärkste Partei, und die LDP rechnete sich gute Chancen aus, dieses auch in Zukunft zu bleiben. Insbesondere die kleineren Parteien fürchteten um eigene politische Positionen und um ihr Überleben. Die SPJ war gespalten. Mehrere Abgeordnete ihres linken Flügels waren entschiedene Gegner der vorgeschlagenen Wahlrechtsreform, da sie in Einerwahlkreisen keine Chance für eine Wiederwahl sahen und auf der Proporzliste der Partei an den Rand gedrängt zu werden fürchteten.

Das entsprechende Wahlrecht zur Wahl des weniger bedeutenden Oberhauses war bereits 1982 geändert worden. Bei den Oberhauswahlen hat jeder Wähler zwei Stimmen, die erste für einen Direktkandidaten im Wahlkreis und eine zweite für die Liste der Parteien. Für die Oberhauswahl entsprechen die 47 Präfekturen Japans je einem Wahlkreis mit einem bis vier Direktmandaten (je nach Bevölkerungszahl der Präfektur). 152 Direktmandaten stehen 100 Listenmandate gegenüber. Die Mitglieder des Oberhauses werden für sechs Jahre gewählt, alle drei Jahre findet die Neuwahl der Hälfte des Oberhauses statt. Im Gegensatz zum Unterhaus kann die zweite Kammer nicht aufgelöst werden. ${ }^{10}$

Zur Bekämpfung der Korruption in der Politik strebte die Regierung eine Änderung der Parteien- und Wahlkampffinanzierung an. Ihr Gesetzentwurf sah ein generelles Verbot von Spenden an einzelne Politiker vor. Diese sollten nur noch an Parteien gehen, die für diesen Zweck aus Gründen der Transparenz nur noch je eine "Spendensammelorganisation" unterhalten durften. Eine Überprüfung war nach Ablauf von fünf Jahren vorgesehen. Die Grenze für die Veröffentlichungspflicht von Parteispenden wurde bei 50.000 Yen festgelegt. Erstmals in der japanischen Geschichte sollten die politischen Parteien aus Steuermitteln alimentiert werden. Der Regierungsentwurf sah dafür pro Kopf der Bevölkerung 335 Yen vor (d.h. insgesamt 41,4 Mrd. Yen), nach einem bestimmten Schlüssel auf die Parteien verteilt. Auch die LDP trat in ihrem Gegenentwurf für eine öffentliche Parteienfinanzierung ein, hielt aber 250 Yen pro Kopf für ausreichend. Die Strafbestimmungen für Verstöße gegen das neue Parteienfinanzierungsgesetz sollten erheblich verschärft werden. Diese sahen insbesondere eine mehrjährige Suspendierung des passiven Wahlrechts vor.

\section{Die politischen Reformen - Ein Kompromiß zwischen Regierung und Opposition}

Premierminister Hosokawa hatte von Anfang an sein politisches Schicksal mit der erfolgreichen Durchsetzung seiner politischen Reformen verknüpft. Angesichts seiner im wesentlichen daraus erwachsenden großen Popularität konnte es sich die in ihrer Mehrheit reformunwillige LDP nicht leisten, die politischen Reformen erneut zu blockieren. Ihr blieben aber viele Möglichkeiten, die Reformen zu verzögern und zu verwässern. Nachdem Hosokawa seinen Gesetzentwurf erfolgreich durch das Unterhaus gebracht hatte, scheiterte 
er überraschend im Oberhaus, weil 17 sozialistische Abgeordnete gegen die Vorlage der eigenen Regierung stimmten. Um das völlige Scheitern seiner Reformen in letzter Minute noch zu verhindern, sah sich Hosokawa zu erheblichen Zugeständnissen an die LDP gezwungen. So gelang es schließlich am 29. Januar 1994, dem letzten Tag der bereits verlängerten Sitzungsperiode, die insgesamt vier Gesetze zur politischen Reform zu verabschieden. Gesetzeskraft erlangte dadurch folgender Kompromiß:

\section{Wahlgesetz}

Einführung eines Mischwahlsystems für das Unterhaus, in dem 300 Abgeordnete direkt in Einerwahlkreisen und 200 in Verhältniswahl bestimmt werden. Aufteilung der Kandidaten bei der Verhältniswahl aus 11 Regionallisten. Jeder Wähler erhält zwei Stimmen. Doppelkandidaturen sind möglich. Die Dauer des Wahlkampfes ist auf 12 Tage festgelegt, wobei erstmals der Haus-zu-Haus-Wahlkampf ("canvassing") zwischen 8.00 und 20.00 Uhr erlaubt ist. 2-Prozent-Sperrklausel bei der Vergabe von Sitzen auf den Regionallisten. Die Stimmenauszählung erfolgt nach dem Höchstzählverfahren von d'Hondt.

\section{Gesetz zur Bestellung eines Ausschusses für die Wahlkreiseinteilung}

Dieser Ausschuß, beim Amt des Premierministers eingesetzt, besteht aus sieben Unterhaus-Abgeordneten und fünf außerparlamentarischen Fachleuten, die von beiden Häusern bestätigt werden. Die Wahlkreiseinteilung erfolgt nach einem Einwohnerschlüssel der Präfekturen.

\section{Gesetz zur Regulierung politischer Spenden}

Direkte Spenden an individuelle Politiker oder politische Gruppierungen sind ausnahmslos verboten. Jeder Politiker darf aber eine Spendensammelorganisation unterhalten, die von einem Unternehmen bis zu 500.000 Yen annehmen kann. Nach Ablauf von fünf Jahren nach Inkrafttreten des Gesetzes werden diese Leistungen (voraussichtlich) verboten. Spenden über mehr als 50.000 Yen müssen unter Namensnennung des Spenders offengelegt werden, ebenso die Namen von Personen, die Karten für sog. "fund-raising-parties" für mehr als 200.000 Yen abnehmen. ${ }^{11}$ Spenden von Einzelpersonen sind steuerlich abzugsfähig. Politische Parteien dürfen durch ihre Spendensammelorganisationen wie bisher Spendenzahlungen von Unternehmen entgegennehmen. Möglich sind je nach Unternehmensgröße Spenden in Höhe von 7,5 Mio. bis 100 Mio. Yen pro Jahr. Zuwiderhandlungen werden mit dem Verlust der bürgerlichen

So gab z.B. im Dezember 1994 der Führer einer der kleineren LDP-Faktionen, Hiroshi Mitsuzuka, eine aufwendige Spendenparty für mehrere tausend Gäste. Von ehemaligen Ministern in seiner Faktion erwartete der Gastgeber, daß sie je tausend der 450 Mark teuren Eintrittskarten an wohlhabende Freunde und Bekannte verkauften (in: Japan - Wirtschaft-Politik-Gesellschaft, Februar 1995, S. 42). 
Ehrenrechte für fünf Jahre bestraft. Dabei gilt der Grundsatz der Mithaftung eines Politikers für die Straftaten seiner Familienmitglieder und engsten Mitarbeiter.

\section{Gesetz zur finanziellen Förderung politischer Parteien}

Insgesamt stehen für die öffentliche Parteienfinanzierung 29,9 Mrd. Yen zur Verfügung, die nach einem Schlüssel aus Zahl der Parlamentssitze und der Stimmenzahl bei nationalen Wahlen auf die Parteien verteilt werden. Um in den Genuß dieser öffentlichen Gelder zu gelangen, muß eine Partei über mindestens fünf Sitze verfügen und bei den vorausgegangenen Unterhaus- (bzw. Oberhaus-)wahlen mindestens 2\% der abgegebenen gültigen Stimmen erhalten haben. Die staatlichen Zuschüsse dürfen aber nicht Zweidrittel der Parteieinnahmen (ohne Kredite) des Vorjahres überschreiten.

Das Kompromißpaket ist zwar nicht der große Wurf, wie ihn Hosokawa und seine Koalition angestrebt hatten, dennoch war damit sechs Jahre nach dem Recruit-Cosmos-Skandal unzweif elhaft ein Durchbruch gelungen. Die LDP setzte sich mit dem angestrebten Übergewicht der Direktmandate gegenüber den Listenmandaten durch. Mit einer größeren Bedeutung der Listenmandate hätten - wie von der Regierung angestrebt - die politischen Parteien erheblich an Bedeutung im politischen Entscheidungsprozeß gewonnen, und der Einfluß der politischen Lokalfürsten wäre zugunsten der Parteizentralen zurückgedrängt worden. Das Übergewicht an Direktmandaten bevorzugt eindeutig die größte Partei. Voraussichtlich wird es sich nur die LDP leisten können, in allen Wahlkreisen Kandidaten mit Aussicht aus Erfolg aufzustellen. Kleinere Parteien müssen sich dagegen auf gemeinsame Kandidaten einigen, was Parteienkooperationen und -zusammenschlüsse begünstigt. Durch die Aufteilung der Listenmandate in elf Regionallisten kommt auch bei der Verhältniswahl der regionale Bekanntheitsgrad eines Politikers zum Tragen (d.h. das traditionelle "Chiban"). Die Reformgesetze scheinen auch nicht dazu geeignet zu sein, die "Politik des großen Geldes" auszumerzen. Es erscheint zweif elhaft, ob für die Nominierung und Wahl eines Kandidaten statt seiner Finanzkraft fortan sein politisches Profil bzw. Programm sowie die sachliche Auseinandersetzung mit dem politischen Gegner ausschlaggebend sein werden. $^{12}$

\section{Rückkehr der Altparteien und Verlangsamung des Reformprozesses}

Premierminister Hosokawa überdauerte seinen Erfolg nur für kurze Zeit. Am 8. April 1994 beugte er sich mit seinem Rücktritt dem Druck der oppositionellen LDP, die jede Parlamentsarbeit blockierte, weil sie unaufhörlich auf die Aufklärung von finanziellen Unregelmäßigkeiten in der Vergangenheit des Premierministers drängte. Am 28. April folgte ihm 
Außenminister Tsutomu Hata im Premierministeramt an der Spitze einer Minderheitsregierung. Die SPJ war nach einem mißlungenen politischen Manöver durch ShinseitoGeneralsekretär Ichiro Ozawa, durch die sie sich an den Rand gedrängt sah, aus der Koalition ausgeschieden. ${ }^{13}$

Ende Juni 1994 wurde die Reformkoalition durch eine Koalition aus LDP, Sozialisten und der kleinen Sakigake abgelöst. Neuer Premierminister wurde Tomoichi Murayama, der Vorsitzende der SPJ und Exponent des linken, reformunwilligen Flügels seiner Partei. SPJ und LDP hatten zwar aufgrund ihrer negativen Einstellung gegenüber den Reformprojekten der bisherigen Regierung zusammengefunden, doch einmal an die Macht zurückgekehrt, hielten auch sie an den eingeleiteten Reformen fest. Der Reformeifer der neuen Regierung war jedoch spürbar geringer. Hosokawa hatte neben der politischen Reform auch eine Reform der Verwaltung und der Wirtschaft (Deregulierung) angestrebt, aber nicht verwirklichen können. ${ }^{14}$

Fristgerecht legte im August der Ausschuß zur Festlegung der Wahlkreisgrenzen seinen Entwurf über den Zuschnitt der 300 Einerwahlkreise vor, der im November nach der Verabschiedung im Unterhaus Gesetzeskraft erlangte. Am 25. Dezember 1994 traten nach ihrer Veröffentlichung im Gesetzesblatt und Ablauf der Ein-Monats-Frist die Reformgesetze endgültig in Kraft. Die nächste Unterhauswahl wird nun endgültig nach dem neuen Wahlrecht stattfinden.

\section{Die Wahlrechtsreform als Motor des politischen Wandels}

Die Wahlrechtsreform wirkt tendenziell auf die Entstehung eines Zweiparteiensystems. ${ }^{15}$ Schon die Aussicht auf die Wahlrechtsänderung hatte bereits Ende 1993 zu einem Zusammenrücken der Parteien geführt. Als erste gingen Shinseito und Komeito eine enge Kooperation ein. Im November 1994 schlossen sich schließlich die bereits im Oppositionsbündnis "Kaikaku" kooperierenden Parteien und Parteigruppierungen ${ }^{16}$ zu einer neuen großen

Vgl. Ichiro Uchida, How Ozawa Blew It, in: The International Economy, Sept/Oct 1994, S. 35-67, S. 62-64.

14 Vgl. Heinricht Kreft, Zeitenwende oder Zwischenspiel? - Die Regierung Hosokawa in Japan, in: Zeitschrift für Politik, 42. Jg. 2, 1995, S. 187-202, S. 197 ff.

15 Ichiro Ozawa, Generalsekretär der Shinseito, trat seit langem offen für ein Zweiparteiensystem nach US-amerikanischem Vorbild ein. Neben die LDP sollte eine weitere in der Grundtendenz ebenfalls konservative Partei treten.

16

Zu diesen gehörten: Shinseito, Komeito, DSP (Demokratisch-Sozialistische Partei, Minshato), JNP, Liberale Partei, Neue Partei Mirai. 
Oppositionspartei zusammen, der "Shinshinto" (Neue Fortschrittspartei) ${ }^{17}$. Mit ihren 214 Abgeordneten wurde die Neugründung zur zweitstärksten Partei im Parlament hinter der LDP (295). Der ehemalige Premierminister Kaifu, der im Juni 1994 gegen Murayama unterlegen war, wurde zum Parteivorsitzenden und Ozawa zu ihrem Generalsekretär gekürt. Beide hatten schon 1990/91 eng zusammengearbeitet, ersterer als Premierminister und letzterer als Generalsekretär der LDP. Nach internen Querelen übernahm Ozawa Anfang 1996 den Parteivorsitz. Bisher sind allerdings die offiziell in der Shinshinto aufgegangenen Parteien keineswegs untergegangen. Ihr Schattenkabinett spiegelt genau die Stärkeverhältnisse der in ihr aufgegangenen ursprünglichen Parteien wider. ${ }^{18}$ Es dürfte noch lange dauern, bis die Shinshinto zu einer homogenen Partei zusammenwächst. Die neue Partei erinnert in vielerlei Hinsicht an die alte LDP. Die Kür des Führungsduos Kaifu-Ozawa war schon im Vorfeld von "Strippenzieher" Ozawa eingefädelt worden, ihre Wahl nur Fassade. Auch das Grundsatzprogramm der neuen Partei ist eher vage gehalten. ${ }^{19}$ Bei den ersten nationalen Wahlen seit der Verabschiedung der Reformgesetze, den Oberhauswahlen vom 23. Juli 1995, schnitt die neue Partei überraschend gut ab und konnte die Zahl der Mandate, die ihre Vorgängerparteien insgesamt innehatten, mehr als verdoppeln. Doch auch dieser Erfolg dürfte die internen Gegensätze nur vorübergehend überdecken. ${ }^{20}$

Auch in der SPJ gab es seit Anfang 1994 Diskussionen über einen Neubeginn, die nach der Bildung der Regierung Murayama zunächst verstummten. Hauptakteur war dabei zunächst der frühere Parteivorsitzende Sadao Yamahana ${ }^{21}$, der Anfang 1995 die Gründung einer innerparteilichen Erneuerungsbewegung anregte, aber nur geringe Resonanz in der SPJ finden konnte. Außer der kleinen Sakigake stehen auch keine Partner zur Verfügung für eine Parteienkooperation oder -fusion. Die tendenziell dafür infrage kommenden Parteien JNP und DSP stehen in Opposition zur Regierung Murayama und haben sich der Shinshinto angeschlossen. Nachdem die SPJ schon der große Verlierer der Unterhauswahlen von 1993 war, erzielte sie in den Oberhauswahlen vom Sommer 1995 das schlechteste Ergebnis ihrer 50jährigen Parteigeschichte. Gegenüber den vorangegangenen Wahlen zur 2. Kammer verlor sie etwa ein Drittel ihrer Mandate. Der Grund für den Niedergang der

17

18

19

20

21

Englisch: "New Frontier Party".

Die Komeito hat sich aus taktischen Gründen gespalten. Während alle Unterhaus- und einige Oberhausabgeordnete der neuen Partei beigetreten sind, blieb die Parteiorganisation nebst den verbleibenden Oberhausabgeordneten und Präfektur- und Kommunalvertretern (vorerst) außerhalb der neuen Partei.

Vgl. Manfred Pohl, Shinshinto: Eine neue Partei mit eingebautem Spaltungsmechanismus, in: Japan - Wirtschaft-Politik-Gesellschaft, Feb. 1995, S. 70-73, S. 71 f.

Vgl. Hijiri Inose, As election euphoria wears off, Shinshinto stumbles, in: Nikkey Weekly, 4.9.1995.

Yamahana trat schließlich im Mai 1995 aus der SPJ aus, fünf weitere Abgeordnete folgten ihm wenig später. 
SPJ dürfte die radikale Abkehr von ihrer traditionellen Politik sein. Als Preis für die große Koalition akzeptierte die SPJ u.a. die Existenz der Streitkräfte und die Sicherheitspartnerschaft mit den USA. Beides hatte sie über Jahrzehnte vehement bekämpft. Es fällt ihr in dieser Situation schwer, sich vor den Wählern als Alternative zu den beiden konservativen Parteien zu profilieren. Im September 1995 hat sie sich zu einem ungewöhnlichen Schritt aufgerafft, der bereits seit Anfang des Jahres diskutiert worden war. Die SPJ löste sich auf, um sich zugleich als "sozial-liberale" Partei - offen für alle liberalen und sozialen Kräfte neuzugründen. Mit diesem Neuanfang hofft sie, ihre Talfahrt zu stoppen und in der neuen Parteienlandschaft als dritte Kraft zu überleben. Seit dem Rücktritt von Premierminister Murayama Anfang Januar 1996 auf Druck der LDP ist nunmehr auch optisch klargestellt, $\mathrm{da} ß$ die Sozialisten in der Koalitionsregierung lediglich den Juniorpartner stellen.

Auch die LDP hat ihr Tief noch nicht überwunden. In den Oberhauswahlen blieb sie mit 49 von 126 zu vergebenden Mandaten deutlich hinter den eigenen Zielen zurück. Doch ihrem neuen Vorsitzenden Ryutaro Hashimoto, der Murayama als Premierminister beerbte, rechnet sie sich gute Chancen aus, in absehbarer Zeit wieder allein die Regierung zu bilden. Hashimotos Popularität war im Sommer noch weiter gestiegen, als er - als Minister für internationalen Handel und Industrie - in einem erst nach erheblicher Eskalation entschärften Handelskonflikt mit den USA $^{22}$ den amerikanischen Unterhändlern erfolgreich Paroli bot. Es ist davon auszugehen, daß die LDP auch in Zukunft eine starke Position im japanischen Parteiensystem behalten wird. Sie verfügt über den besten Parteiapparat und ein erhebliches Spendenaufkommen.

Die Wahlrechtsreform macht neue Wahlkampfstrategien erforderlich und wird die Herausbildung eines neuen Politiker-Typus begünstigen. Das größere Gewicht der Einerwahlkreise im Vergleich zu den Verhältniswahlbezirken zwingt die Kandidaten und die hinter ihnen stehenden Parteien, ihre programmatischen Besonderheiten in den Vordergrund ihres Wahlkampfes zu stellen. Dieses führt tendenziell zu klareren Alternativen für den Wähler und zu einer neuen Politik, die sich stärker an der Schaffung öffentlicher Güter anstatt an der Umverteilung zugunsten von Partikularinteressen orientiert. Insgesamt dürfte sich die Intensität des politischen Wettbewerbs zwischen den Kandidaten und den hinter ihnen stehenden Parteien verstärken. Die Kandidaten müssen ihre Kontakte zu den Wählern verstärken und auch bisher vernachlässigte Wählergruppen ansprechen. Dadurch könnte ein Mobilisierungseffekt ausgelöst werden, der sich kurzfristig positiv auf die Wahlbeteiligung und längerfristig auf die Partizipation der Bürger insgesamt auswirkt. ${ }^{23}$ Wie dringend notwendig diese Reformen sind, zeigt der stete Rückgang der Wahlbeteiligung. Bei den

Vgl. Heinrich Kreft, Japan - USA: Primat der Handelsbeziehungen?, in: Außenpolitik II 1995, S. 175-185.

23

Vgl. Paul Kevenhörster, Das japanische Parteiensystem im Umbruch. Stagnation oder Neubeginn?, in: Aus Politik und Zeitgeschichte, B 50/94, S. 3-10, S. 10. 
Oberhauswahlen im Juli 1995 lag die Wahlbeteiligung mit 44,52\% weit unter dem bisherigen Minusrekord von 50,07\% in den vorangegangenen Oberhauswahlen von 1992.

Der innenpolitische Reformprozeß, der vor drei Jahren in Gang gesetzt wurde, ist noch nicht zum Abschluß gekommen. Das Parteiengefüge hat noch keine neue Stabilität gefunden. Die nächsten Unterhauswahlen, die spätestens im Sommer 1997 stattfinden müssen, dürften den vorläufigen Abschluß dieses Reformprozesses bilden.

Wie andere westliche Industriestaaten steht auch Japan vor einer Fülle von Herausforderungen in unterschiedlichen Politikbereichen, wie Deregulierung der Wirtschaft, Verschlankung des Staates, Bekämpfung der Arbeitslosigkeit, Gewährleistung der sozialen Sicherung, Steuerreformen und eine zeitgemäße Bildungspolitik sowie im internationalen Bereich die Übernahme von größerer Verantwortung (u.a. VN-Sicherheitsratssitz, Beteiligung an VN-Friedensmissionen). ${ }^{24}$ Zur Bewältigung dieser Aufgaben benötigt Japan heute mehr denn je eine entschlossene Führung, die die große Mehrheit der Bevölkerung hinter sich weiß. 
The paper examines the formulation and implementation of the programme to return the country to constitutional rule, with special emphasis on the problems encountered and their implications for the building of a democratic culture in Ghana. First, it examines the design of the programme to return the country to constitutional rule. It then proceeds to analyze the strategies and procedures devised for the realization of the programme. Lastly, the paper discusses the elections, the performance of the civilian government and prospects for the future.

\title{
Political Reform in Japan
}

\section{By Heinrich Kreft}

The author analyses the decline of the Liberal Democratic Party (LDP) in the wake of the inauguration of the first non-LDP government in 1993. Corruption and scandals made a political reform inevitable, but the opposition parties (mainly the Socialist Party) because of their internal weaknesses did not pose a serious challenge to the ruling LDP. Political reform became possible only when the LDP split and its reform-minded faction formed a coalition with smaller conservative parties and the socialists. After fierce battles with the LDP the new government finally succeeded in reforming the electoral system for the Lower House. Under the new law multi-member constituencies will be abandoned for the next election, due by 1997. Candidates for parliament will run for 300 single-member constituencies; 200 additional seats are filled by proportional representation. This reform could fundamentally change Japan's political landscape. Even though the reform-government of Prime Minister Hosokawa and its successor had been replaced already in 1994 by a coalition of the LDP and the Socialist Party, the reform process was only slowed down but not halted. The next parliamentary elections will thus be crucial for the political reform process in Japan.

\section{The Granting of Amnesty for Human Rights Violations in Latin America from an International Criminal Court Perspective}

\author{
By Kai Ambos
}

One of the most frequent reasons for the impunity (impunidad) of human rights violations in Latin America is the granting of amnesty in favor of perpetrators - who are often mem- 